\title{
HISTOLOGIC PARAMETERS FOR STUDYING MULTIPLE BLOOD MEALS IN AEDES ALBOPICTUS (SKUSE) (DIPTERA: CULICIDAE)
}

\section{By}

ABEER S. YAMANY ${ }^{1}$ FATMA K. ADHAM ${ }^{2}$ AND HEINZ MEHLHORN ${ }^{3}$

Department of Zoology, Faculty of Science, Zagazig University ${ }^{1}$, Zagazig, Entomology of Department, Faculty of Science, Cairo University ${ }^{2}$, Giza, Egypt, and Institute of Zoology, Cell Biology and Parasitology ${ }^{3}$, Heinrich-Heine University, Duesseldorf, Germany (*Correspondence: emarewan@yahoo.com)

\section{Abstract}

During a single gonotrophic cycle, histologic technique was used to detect the multiple blood feedings in Ae. albopictus. During a 3-day period, 200 blood engorged Ae. albopictus mosquito females were examined. The results showed that $69 \%(138 / 200)$ of these females imbibed two blood meals. Time between the second blood meal and fixation ranged from 0 to $32 \mathrm{~h}$. When applying histologic parameters, only $23 \%$ of females with multiple meals were detected. Our data suggest that the limit for histologic detection of multiple feeding were more restricted for Ae. albopictus. Histological studies revealed the presence of an early blood meal within the second one appearing as a body of dark digested blood mixed with heme $(\mathrm{H})$, or sometimes a pink undigested blood associated with a bluestaining peritrophic plug (PP) surrounding the blood meal and the presence of a physical separation (PS) separating the blood meals. When using histologic technique of field collected mosquitoes, advanced stages of oocytes or development of ovarian follicles with apparent dilations were observed.

Key words: Aedes albopictus, multiple blood feeding, histology.

\section{Introduction}

In most species of mosquitoes, ingestion of one blood meal initiates egg development. In epidemiological studies the biting behavior of female mosquitoes may be very important in the study of disease transmission. Moreover, host contacts of mosquitoes during the ingestion of blood might increase the possibility of acquiring and transmitting viral pathogens. During each gonotrophic cycle, multiple blood feeding in laboratory mosquitoes was reported (Tempelis, 1975), which were reported in the Aedes aegypti (Chow et al, 1993; Scott et al, 1993); in Culiseta melanura (Lorenz and Scott, 1996) and in Anopheles freeborni and Culex tarsalis (Wekesa et al, 1997). The host choice and multiple blood feeding behavior of malaria vectors and other anophelines were studied by Muriu et al. (2008) and Xue at al. (2009) in Ae. albopictus. The identification of multiple blood meals in mosquitoes during one gonotrophic cycle using histological techniques was reported by Romoser et al. (1989).

In Egypt, Aedes aegypti was reported in the Toshka district (Shoukry et al, 2012) and southern Aswan (Saleh, 2012).The Aedes-borne diseases as Dengue, Chikungunya, Zika, and Yellow fever are an emerging problem worldwide, particularly encountered in Africa (Heikal et al, 2011). ElBahnasawy et al. (2011) reported that the presence of Ae. aegypti, as the rapid population growth rural-urban migration and the increase in volume of solid waste as well as endemicity of dengue and dengue hemorrhagic fevers in the neighboring regional countries being threat to Egypt from travelers. Dengue fever cases have been registered earlier in Egypt; the last outbreak was in 2015 in Dairut (WHO, 2015). Saifullin et al. (2018) in Russia reported that in 2017, two cases of dengue fever were imported from Hurghada, Egypt Ducheyne et al. (2018) gave maps for the distribution and maximum risk created for Ae. aegypti and Ae. albopictus for the WHO/ EMRO. These region-speci-fic maps highlighted data gaps which they filled using targeted monitoring and surveillance.

The present study aimed at following the 
procedure and criteria described by the previously mentioned authors was applied to study the frequency feeding during one gonotrophic cycle among wild mosquitoes Aedes albopictus of dengue fever (DEN) using a standard histologic technique.

\section{Materials and Methods}

Aedes albopictus used during this study were obtained from a laboratory colony maintained for more than 15 generations in the department of Parasitology, Cell biology and Zoology Institute, Heinrich Heine University, Düsseldorf, Germany. Mosquitoes were maintained under laboratory conditions of $25 \pm 2^{\circ} \mathrm{C}, 70-80 \%$ R.H. \& $12 \mathrm{hr}$ light: $12 \mathrm{hr}$ night photoperiod. Newly emerged adult females were supplied with $10 \%$ sucrose and removed $>6 \mathrm{hr}$ before Guinea pig exposure. In most cases, the first blood meal was interrupted. Mosquitoes were kept in the standard laboratory conditions, were collected between meals and after the second one during the afternoon from the chamber. The histologic technique applied during this study was similar to that used in the institute where the Ae. albopictus mosquitoes obtained with some modifications. Sedation of mosquitoes was achieved by placing them on wet ice. Legs, thorax and last three abdominal segments were carefully removed with forceps to facilitate the penetration of fixative. Specimens were fixed in $2.5 \%$ Gluteraldhyde in 0.1 Cacodylate buffer solution ( $\mathrm{pH}$ 7.4) and kept for 7 days in the refrigerator $\left(-7^{\circ} \mathrm{C}\right)$, followed by 4 changes for one hour in the Cacodylat buffer (better if tannin were used) and then specimens were dehydrated in ascending series of alcohol, followed by two volumes of Propylenoxide \& one volume of Epon and finally in one volume of Propylenoxide $\&$ two volumes of Epon overnight. Then kept in fresh Epon (in desiccators) for 24hr, then finally embedded in fresh Epon for $48 \mathrm{hr}$ at $40^{\circ} \mathrm{C}$ then for $24 \mathrm{hr}$ at $60^{\circ} \mathrm{C}$ (Luft, 1961), and stored at $4^{\circ} \mathrm{C}$ for at least 5-7 days. The sections, 5-8 um thick, were stained with methyl blue. Detection of mul- tiple blood meals imbibed by mosquitoes was used (Romoser et al, 1989). These were: peritrophic membrane (PM), peritrophic plug $(\mathrm{PP})$, heme $(\mathrm{H})$ and physical separation (PS). During the study, a new parameter was added the ovarian development, which define as a mosquito with fresh red blood meal and oocytes in stage 3 to stage 7 of Christopher's stages of ovarian development (Christopher, 1960). The dissection of the ovaries was avoided due to the small size, and thus this has the advantage of having the ovaries held in position by the viscera.

\section{Results}

Under standard laboratory conditions, 138 Aedes albopictus mosquitoes imbibed the second blood meal after a certain time of the first one were examined and fixed (Tab. 1). Data showed that 1- multiple meals detected in $91 \%(21 / 23)$ when mosquitoes were fixed immediately after the second meal. 2- when two meals were separated by $8-48 \mathrm{hr}$ and fixed $0-8 \mathrm{hr}$ after the second meal, $78 \%(56 / 72)$ of the known double meals were detected, but $44 \%(29 / 66)$ of the multiple meals were detected outside this range. A physical separation between meals with a line of amorphous material and the amount of heme that developed around each meal was detected within $62 \%(85 / 138)$. 3When interval between meal was brief, a line of amorphous, reddish-brown, yellowish brown or blue-staining was detected. Based on immunohistochemical studies, this area was plasma. The (PM) of these mosquitoes took the form of a fluid secretion. Blood bolus cells were compressed when time between meals, plasma was extruded to the periphery of the bolus Perrone and Spielman (1988), (Figs. 1, 2, 3, 4 \& 9) showed a clear space between meals. $4-$ Detectable multiple meals observed in $27 \%$ $(4 / 15)$ of the mosquitoes when meals were separated by $56 \mathrm{hr}$. In $59 \%$ (10/17) of those mosquitoes, the first meal was no longer detectable $64 \mathrm{hr}$ after their first blood meal and $47 \%(7 / 15)$ contained double meals. 
Thirty-two hours after their second blood meal which were separated from the first blood meal by $16 \mathrm{hr}$ showed the highest percentage $82 \%(14 / 17)$ of the specimens containing detectable double meals. While the lowest percentage $39 \%(9 / 23)$ of the specimens containing detectable blood meals when fixed $32 \mathrm{~h}$ after second blood meal.

The 21\% (19/92) of the specimens imbibed their last blood meal $24 \mathrm{~h}$ before collected; $57 \%$ (52/92) and 20\% (18/92) a day and $32 \mathrm{hr}$ before being collected, respectively (Tabs 2 \& 3). Histologically, multiple meals were detected in $41 \%$ (38/92) of Ae. albopictus examined that had imbibed two meals and 59\% (54/92) imbibed one blood meal. Most mosquitoes had fed blood meal on consecutive days was $27 \%$ (17/64), $59 \%$ (38/64) and 14\% (9/64) imbibed two blood meals on the same day, consecutive days and separated by $32 \mathrm{hr}$, respectively. Second meal was taken during the same day in $30 \%$ (3/10) of mosquitoes, on consecutive days in $60 \%(6 / 10)$ and $32 \mathrm{hr}$ in $10 \%(1 / 10)$.

Mosquitoes which fed multiple meals, heme around the first meal $(98 \%, 48 / 49)$ and a physical separation between meals $(69 \%$, 34/49) were the most useful parameters for determining that more than one meal had been imbibed (Figs. 1, 2, 3, 4, 5, 6 and 9). Ovarian development was used to detect multiple feeding as in 39\% (19/49) and a fully digested meal surrounded by a recent red meal was detected in $49 \%$ (24/49) of the mosquitoes (Tab.4). While results in table 5 showed that $43 \%(35 / 81)$ of mosquitoes that imbibed one meal had oocyte in stage 1 to stage 3 , but $57 \%(46 / 81)$ had oocyte in stage 4 to stage 7 . Conversely, $80 \%$ (68/85) of mosquitoes had oocytes in stage 4 to stage 7 , while $20 \%$ (17/85) had oocyte in stage 1 to stage 3 . Mosquitoes that fed three blood meals had oocyte in stage 4 to stage 7. When the interval between blood meals was $16 \mathrm{~h}$ and between the second blood meal and the fixation was $0-6 \mathrm{hr}$ a heme $(\mathrm{H})$ separate between the first and second meals was detected. The second meal is a fresh blood stained with methylene blue. Ovary was in the third stage indicated by an increase in the size of primary follicle (F1) with the follicular epithelium (FE) with a large nucleus, oocyte was enlarged, the lipid droplets became visible in the cytoplasm of oocyte and some small particles of yolk were deposited, as a few numbered yolk granules surrounded the nucleus. The oocyte nucleus was spherical and surrounded by a yolk-free cytoplasm, meanwhile the NC occupied the remnant two thirds. The cytoplasm of each $\mathrm{NC}$ appeared granular. The nuclear membrane and nucleolus of the oocyte's nucleus were distinct. The GA in the NC underwent a very tight clumping (Fig.1 and 9). Some of the collected specimens fixed after 0-6hr appeared with the histologic technique with only one blood meal as there was no any indication of previous blood meal as the ovary was in the stage 2 of development (Fig. 8). In the contrary there were other specimens fixed 0-6 h after collection appeared with the histologic technique with only one blood meal meanwhile the ovary was in the stage 3 of the development indicating that there was a previous small or interrupted blood meal which was digested completely and separated from the second meal by $56 \mathrm{hr}$ (Fig. 7).

As the interval between the blood meals increased to 24-32hr and to $8 \mathrm{hr}$ (Fig 2) between the second blood meal and the fixation, the ovary was in the stage 4 showed one or two rows of yolk granules surrounding the nucleus and increasing until the cytoplasm was filled with yolk. Meanwhile the follicle increased in size, and became ovoid, the FE was provided with a large nucleus and a small amount of cytoplasm. The NC occupied about half of the egg follicle and the oocyte increased in size occupying the other half. Furthermore, the ON contained a central mass of $\mathrm{CH}$. The number of the follicular epithelial cells (FE) increased. The lipid droplets became visible as well as a yolk deposition. The mitochondria increased in number and the GA in the 
NC appeared as small granules (Fig. 2).

As the interval between the blood meals increased to $40 \mathrm{hr}$ and time after the second blood meal increased to $16 \mathrm{hr}$, ovary appeared in stage 5 as the follicles increased in size and NC nuclei reached their maximum size. Yolk spheres increased in number and size hiding the nucleus of the oocyte. Large densely packed yolk spheres Y1 and small ones Y2 filling about three fourths of the oocyte (Fig. 3). Epithelial cells surrounding the NC became enlarged, while those surrounding the oocyte were still large, cuboidal, and were separated from each other by intercellular spaces. Vitelline membrane (VM) that adhered to both the oocyte and FE now became visible but only secreted when yolk formation was completed (Fig. 3 ). When a mosquito imbibed two blood meals separated by $56 \mathrm{hr}$ and from the fixation by $16 \mathrm{hr}$, (Fig. 4) there was a dark line of heme around the previous small or interrupted first blood meal which was nearly digested. The second meal was fresh stained with methylene blue. Ovary appeared as in stage 2 because the first blood meal was very small and not enough to start the development of the ovary which remain in stage 2 showed that the primary follicle (F1) was completely separated from germanium. Oocyte was in the distal portion of the follicle. Seven NC were seen above the oocyte. The NC appears relatively larger containing a low amount of cytoplasm. Very small refractile lipid droplets were in ooplasm. Ovary appeared when the intervals between the first and the second blood meal were $48 \mathrm{hr}$ and $24-32 \mathrm{hr}$ between second blood meal and fixation indicated by oocyte which underwent growth, increase in size, occupying nine tenth of the follicle. The ON was formed at extreme anterior end and just below the VM and the NC. Continuous VM was formed as a thick layer. There were no follicular epithelial cells FE between the NC and the oocyte (Fig. 5). When the interval between the first and second blood meals increased to $56 \mathrm{hr}$ and the time after the second meal to the fixation was $24-32 \mathrm{hr}$ (Fig.6) indicated by the ovary which was in stage 7 of development as there were a well-developed egg, degeneration of the NC. Three main regions were detected in the mature egg: yolk mass, the VM, and the chorion. The follicular epithelial cells disappeared. There was a secretion of the chorion, which composed of an inner layer, (1) the endochorion (ENC), which was narrow homogeneous and composed of two layers and (2) the exochorion (EXC), composed of several layers. Details are given in tables (1, 2, 3, 4 \& 5).

Table 1: Determination of the two blood meals imbibed by Ae. albopictus $(\mathrm{n}=138)$.

\begin{tabular}{|c|c|c|c|c|c|c|}
\hline \multirow{2}{*}{$\begin{array}{c}\text { Time from first meal to } \\
\text { second one (hr) }\end{array}$} & \multicolumn{5}{|c|}{ Time between the second meal and fixation (hr) } \\
\cline { 2 - 7 } & 0 & 6 & 8 & 16 & 24 & 32 \\
\hline 8 & $3 / 3$ & $2 / 3$ & $1 / 3$ & $2 / 3$ & $0 / 2$ & $2 / 3$ \\
\hline 16 & $4 / 4$ & $3 / 3$ & $2 / 3$ & $3 / 4$ & $0 / 1$ & $2 / 2$ \\
\hline 24 & $4 / 5$ & $5 / 5$ & $4 / 4$ & $2 / 4$ & $2 / 4$ & $3 / 4$ \\
\hline 32 & $6 / 6$ & $4 / 6$ & $3 / 5$ & $3 / 4$ & $3 / 6$ & $2 / 4$ \\
\hline 48 & $1 / 1$ & $2 / 2$ & $0 / 2$ & $2 / 2$ & $4 / 5$ & $0 / 5$ \\
\hline 56 & $1 / 2$ & $0 / 2$ & $2 / 3$ & $0 / 3$ & $1 / 2$ & $0 / 3$ \\
\hline 64 & $2 / 2$ & $2 / 2$ & $3 / 3$ & $0 / 3$ & $0 / 3$ & $0 / 2$ \\
\hline
\end{tabular}

Table 2: Intervals from the recent blood meal and capture for 92 Ae. albopictus.

\begin{tabular}{|c|c|c|c|c|}
\hline \multirow{2}{*}{ No. blood meals per gonotrophic cycle } & \multicolumn{5}{|c|}{ Time from the last blood meal and capture } \\
\cline { 2 - 5 } & on day & $24 \mathrm{hr}$ & $32 \mathrm{hr}$ & Total \\
\hline one meal & 13 & 28 & 13 & 54 \\
\hline Two meals & 9 & 24 & 5 & 38 \\
\hline Three meals & 0 & 0 & 0 & 0 \\
\hline Total & 19 & 52 & 18 & 92 \\
\hline \% of total & 21 & 57 & 20 & \\
\hline
\end{tabular}


Table 3: Intervals between blood meals fed by 86 Ae. albopictus.

\begin{tabular}{|c|c|c|c|c|}
\hline \multirow{2}{*}{ No. blood meals per gonotrophic cycle } & \multicolumn{4}{|c|}{ Interval between multiple meals } \\
\cline { 2 - 5 } & Same day & $24 \mathrm{hr}$ & $32 \mathrm{hr}$ & Total \\
\hline Double meal & 17 & 38 & 9 & 64 \\
\hline Triple meal & 0 & 0 & 0 & 0 \\
\hline First meal & 2 & 3 & 7 & 12 \\
\hline Second meal & 3 & 6 & 1 & 10 \\
\hline Total & 22 & 47 & 17 & 86 \\
\hline
\end{tabular}

Table 4: Frequency of detection of histologic parameters in 98 Ae. Albopictus per gonotrophic cycle.

\begin{tabular}{|c|c|c|c|c|c|}
\hline $\begin{array}{c}\text { No. blood } \\
\text { meals }\end{array}$ & PM $^{a}$ & Heme & $\begin{array}{c}\text { Fully } \\
\text { digested }\end{array}$ & $\begin{array}{c}\text { Physical } \\
\text { separation }\end{array}$ & $\begin{array}{c}\text { Ovarian } \\
\text { development }\end{array}$ \\
\hline Single meal & 0 & 46 & 16 & 0 & 0 \\
\hline Double meal & 17 & 48 & 24 & 34 & 19 \\
\hline Total & 17 & 94 & 40 & 34 & 19 \\
\hline$\%$ of total & 17 & 96 & 41 & 35 & 20 \\
\hline
\end{tabular}

${ }^{a}$ Peritrophic membrane or peritrophic plug Discussion:

Table 5: Christophers̉ stage of ovarian development for 166 Ae. albopictus.

\begin{tabular}{|c|c|c|c|c|c|c|c|c|}
\hline \multirow{2}{*}{$\begin{array}{c}\text { No. blood meals per } \\
\text { gonotrophic cycle }\end{array}$} & \multicolumn{9}{|c|}{ Stage of ovarian development } \\
\cline { 2 - 10 } & Stage 1 & Stage 2 & Stage 3 & Stage 4 & Stage 5 & Stage 6 & Stage 7 & Total \\
\hline Single meal & 4 & 8 & 23 & 21 & 12 & 9 & 4 & 81 \\
\hline Double meals & 0 & 2 & 15 & 26 & 23 & 10 & 9 & 85 \\
\hline Total & 4 & 10 & 38 & 47 & 35 & 19 & 13 & 166 \\
\hline \% of total & 2 & 6 & 23 & 28 & 21 & 11 & 8 & \\
\hline
\end{tabular}

Discussions

In the present study, the limits for histologic detection of multiple feeding were more restricted for Ae. albopictus than in case of $C x$. nigripalpus and Ae. aegypti. At $25^{\circ} \mathrm{C} 80 \%$ of the known multiple meals were detected when feeding was separated by 1 to $\leq 24 \mathrm{hr}$ and were fixed $\leq 12 \mathrm{hr}$ after the second meal Scott et al. (1993).

In case of 333 blood engorged An. freeborni and $41 C x$. tarsalis females and revealed that 13 and $10 \%$ of these females imbibed multiple blood meals, respectively Wekesa et al. (1997).

In the present study, where Ae.albopictus were held at $25 \pm 2^{\circ} \mathrm{C}, 70-80 \%$ R.H. \& $12 \mathrm{hr}$ light: $12 \mathrm{hr}$ night photoperiod, only $78 \%$ of the known multiple meals were detected when feeding was separated by 8 to $\leq 24 \mathrm{hr}$ and mosquitoes were fixed $\leq 16 \mathrm{hr}$ after the $2^{\text {nd }}$ meal. The difference between the present results and those of Romoser et al. (1989) and Scott et al. (1993) might be due to interspecific differences; i.e., the rate of blood meal digestion might be faster in $A e$. albopictus than in $C x$. nigripalpus and Ae. aegypti. Another consideration was that the present sample size was larger (138 vs. 58) than theirs. The female engorged Ae. albopictus $(41 \%)$ examined were fed on two blood meals while 59\% (54/92) of specimens had fed single blood meal were detected. Although this estimate was within the range reported for Ae. albopictus attracted to human bait and examined externally in other parts of the world (42\% in Malaysia (Macdonald, 1956), 72\% in Thailand (Yasuno and Tonn, 1970), and 51\% in Kenya (Trpis and Hausermann, 1986), 50\% in San Juan, Puerto Rico (Scott et al, 1993). The present results agreed with Scott et al. (1993) for Ae. aegypti. The histologic technique is to detect more than one meal separated by $1-2 \mathrm{~d}$ or less and fixed $\leq 24 \mathrm{hr}$ after second meal for Ae. albopictus. Most mosquitoes that contained more than one blood meal had fed blood on consecutive days. $27 \%(17 / 64)$ imbibed two meals on the same day; 59\% (38/64) imbibed two meals on consecutive days; and $14 \%(9 / 64)$ fed twice separated by $32 \mathrm{hr}$. All mosquitoes fed twice had taken their first meal $32 \mathrm{hr}$ before the last one. $30 \%(3 / 10)$ of mosquitoes had fed the second meal on same day, $60 \%$ $(6 / 10)$ on consecutive days and $10 \%(1 / 10)$ at least $32 \mathrm{hr}$. 
Wekesa et al. (1995; 1997) studied the multiple blood feeding in Anopheles freeborni and found that the frequency of multiple blood feeding in field populations of An. freeborni could be determined. Fifty-six laboratory-reared mosquitoes were offered two blood meals and were used to define the limits of the histologic procedure we applied during our study $78 \%$ of known double meals were detected when the interval between meals was from 1 to $24 \mathrm{~h}$, and the time from the second meal until fixation ranged from 0 to $24 \mathrm{hr}$. At intervals outside this range, $50 \%$ of multiple meals were detected. The most important histologic parameters for determination of multiple feeding in field-collected An. freeborni were the amount of heme that developed around each meal, the peritrophic membrane, partially digested blood meals, and physical blood meal separation. Examination of 134 bloodengorged An. freeborni collected in the field showed that $9.7 \%$ had imbibed multiple blood meals. These results suggested that multiple blood meals in An. freeborni were more frequent than previously thought, with significant epidemiologic implications.

Wekesa et al. (1997) confirmed that multiple blood meals in An. freeborni, and perhaps in $C x$. tarsalis, were a frequent phenomenon, and that the histologic procedure was an appropriate tool for longitudinal and region-wide detection of multiple blood feeding necessary in epidemiologic studies. Aedes aegypti imbibes multiple blood meals in each gonotrophic cycle (Scott et al, 1993). It prefers human blood, and rarely feeds on sugar (Edman et al, 1992) and (Van Handel et al, 1994). The host seek by the Ae. aegypti females have bodies of widely differing sizes, and exhibit variable blood feeding frequencies (Nasci, 1986). Smallest females often engage in more multiple feeding than largest ones (Nayar and Sauerman, 1975). This metabolic adjustment was compensated by remaining previtellogenic in the Christopher's stage I-IIb (MacDonald, 1956), hence they required a subsequent blood meal to become vitellogenic beyond stage 3 and produced mature eggs (Clements, 1992). Egg development may require multiple blood meals among small Aedes aegypti field collected in northeastern Mexico (Villanueva, 2004).

\section{Conclusion}

Generally speaking, there are increase in the awareness and preparedness of the different countries for Aedes borne diseases. Undoubtedly, the histological studies of the any mosquito-vector would be useful and of great value, helping in evaluating the blood feeding frequency of field-caught tiger mosquitoes Ae. albopictus in endemic areas of viral pathogens

\section{References}

Chow, ER, Wirt, A, Scott, TW, 1993: Identification of blood meals in Aedes aegypti by antibody sandwich enzyme-linked immunosorbent assay. J. Am. Mosq. Control Assoc. 9:196-205.

Christopher, RS, 1960: Christopher's (1960) Aedes aegypti (L.) the yellow fever mosquito. Cambridge University Press, London.

Clements, AN, 1992: Vitellogenesis, the Biology of Mosquitoes, Development, Nutrition and Reproduction. V. 1, Chapman \& Hall, London. Ducheyne, E, Tran Minh, NN, Haddad, N, Bryssinckx, W, Buliva, E, et al, 2018: Current and future distribution of Aedes aegypti and Aedes albopictus (Diptera: Culicidae) in WHO Eastern Mediterranean Region. Int. J. Hlth. Geogr. Feb 14;17(1):4. doi: 10.1186/s12942-0180125-0.

Edman, JD, Strickman, D, Kittayapong, P, Scott, TW, 1992: Female Aedes aegypti (Diptera: Culicidae) in Thailand rarely feed on sugar. J. Med. Entomol. 29:443-74.

El-Bahnasawy, MM, Khalil, HH, Morsy, AT, Morsy, TA, 2011: Threat of dengue fever and dengue haemorrhagic fever to Egypt from travelers. J. Egypt. Soc. Parasitol. 41, 2:289-306.

Heikal, OM, El-Bahnasawy, MM, Morsy, A T, Khalil, HH, 2011: Aedes aegypti re-emerging in Egypt: a review and what should be done? J. Egypt. Soc. Parasitol. 41, 3:801-14.

Lorenz, LH, Scott, TW, 1996: Detection of multiple blood feeding in Cx. melanura. J. Am. Mosq. Control Assoc. 12:135-6.

Luft, JH, 1961: Improvements in epoxy resin embedding methods. J. Biophys. Biochem. Cyt- 
ol. 9: 409-10.

Macdonald, WW, 1956: Aedes aegypti in Malaya. II. Larval and adult biology. Ann. Trop. Med. Parasitol.50: 399-414.

Muriu, SM, Muturi, EJ, Shililu, JI, Mbogo, CM, Mwangangi, JM, et al, 2008: Host choice and multiple blood feeding behavior of malaria vectors and other anophelines in Mwea Rice Scheme, Kenya. Malar. J. 7, 43:1-7.

Nasci, RS, 1986: The size of emerging small and host seeking Aedes aegypti and the relationship of size to blood feeding success in the field. J. Am. Mosq. Control Assoc. 2:61-2.

Nayar, JK, Sauerman, DM, 1975: The effects of nutrition on survival and fecundity in Florida mosquitoes. Part 3- Utilization of blood and sugar for fecundity. J. Med. Entomol. 12:220-5.

Perrone, JB, Spielman, A, 1988: Time and site of assembly of the peritrophic membrane of the mosquito Aedes aegypti. Cell Tissue Res. 252: 473-8.

Romoser, WS, Edman, JD, Lorenz, LH, Scott, TW, 1989: Histological parameters useful in the identification of multiple blood meals in mosquitoes. Am. J. Trop. Med. Hyg. 41:737-42. Saleh, NM, 2012: Aedes mosquito in Aswan Governorate, Egypt. J. Egypt. Soc. Parasitol. 42, 1:233-8.

Saifullin, MA, Laritchev, VP, Grigorieva, Y E, Zvereva, NN, Domkina, AM, et al, 2018: Two cases of dengue fever imported from Egypt to Russia, 2017. Emerg Infect Dis. Apr 17;24(4). doi: 10.3201/eid2404.172131.

Scott, TW, Clark, GG, Lorenz, LH, Amerasingher, PH, Reiter, P, et al, 1993: Detection of multiple blood feeding in Aedes aegypti (Diptera: Culicidae) during a single gonotrophic cycle using a histologic technique. J. Med. Entomol. 30, 1:94-9.

Shoukry, NM, Elwan, MA, Morsy, TA, 2012:
Aedes aegypti (Linnaeus) re-emerging in southern Egypt. J. Egypt. Soc. Parasitol. 42, 1:41-50.

Tempelis, CH, 1975: Host feeding patterns of mosquitoes, with a review of advances inanalysis of blood meals by serology. J. Med. Entomol. 11:635-53.

Trpis, M, Hausermann, W, 1986: Dispersal and other population parameters of Aedes aegypti in an African village and their possible significance in epidemiology of vector-borne diseases. Am. J. Trop. Med. Hyg. 35:1263-79.

Van Handel, EJ, Edman, JD, Day, JF, Scott, TW, Clark, GC, et al, 1994: Plant-sugar, glycogen and lipid assay of Aedes aegypti collected in urban Puerto Rico and rural Florida. J. Am. Mosq. Control Assoc. 10:149-53.

Villanueva, FR, 2004) Egg Development May Require Multiple Blood meals among Small Aedes aegypti (Diptera: Culicidae) Field Collected in Northeastern Mexico. Florida Entomol. Soc. 87, 4: 630-2.

Wekesa, JW, Yuval, B, Washino, RK, 1995: Multiple blood feeding in Anopheles freeborni (Diptera: Culicidae). Am. J. Trop. Med. Hyg. 52, 6:508-11.

Wekesa, JW, Yuval, B, Washino, RK, 1997: Multiple blood feeding by Anopheles freeborni and Culex tarsalis (Diptera: Culicidae): Spatial and temporal variation. J. Med. Entomol. 34, 2: 219-25.

WHO, 2015: Dengue fever-Egypt [cited 2017 Dec 11]. http://www.who.int/csr/don/12-novem ber-2015- dengue/en/External Link

Yasuno, M, Tonn, RJ, 1970: A study of biting habits of Aedes aegypti in Bangkok, Thailand.

Bull. WHO 43:319-25

Xue, R, Bamard, DR, Ali, A, 2009: Influence of multiple blood meals on gonotrophic dissociation and fecundity in Aedes albopictus. J. Am. Mosq. Control Assoc. 25, 4:504-7

\section{Explanation of figure}

Fig. 1: Light micrographs.:Semithin histologic longitudinal sections (8um) of engorged Aedes albopictus. Abbreviations denote the following: BM1, first blood meal; BM2 second blood meal; PS, physical separation; PM, peritrophic membrane. A mosquito that fed twice separated by $16 \mathrm{~h}$ and time after second blood meal to fixation $0-6 \mathrm{hr}$. Second meal stained with methylene blue (a), by $24 \mathrm{hr}$ (b). (c, d) ovary in third stage showed that F1, primary follicle; FE, follicular epithelial cell; G, germarium; NC, nurse cell; NCN, nurse cell nucleus; O, oocyte; ON oocyte nucleus, OLS, ovariolar sheath; OVI oviduct; Y1, large yolk spheres; Y2, small yolk spheres, 


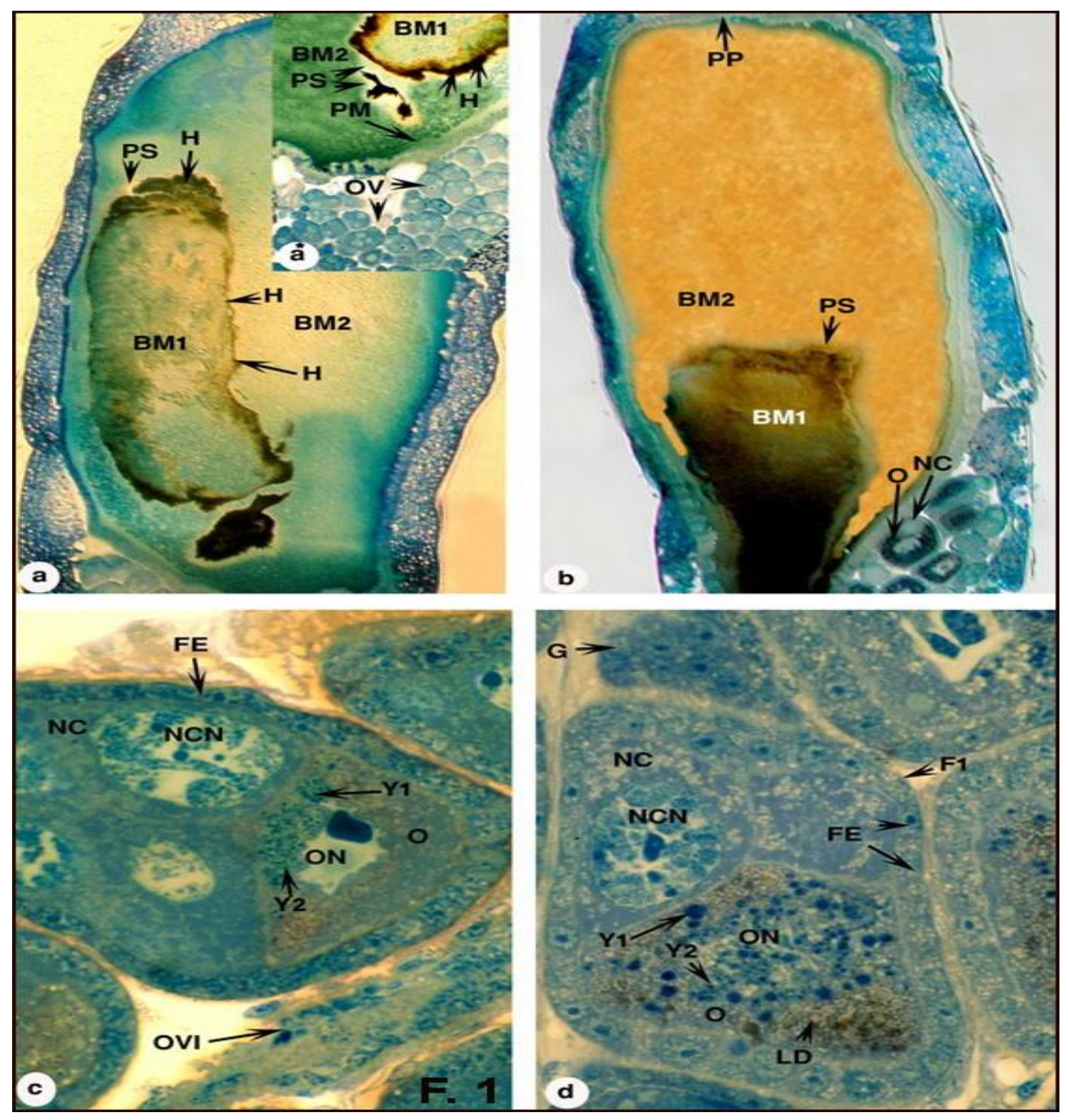

